\title{
Review
}

Jin Zhang*

\section{Research progress on the role of immune cells in Brucella infection}

DOI: $10.2478 / \mathrm{ii}-2018-0014$

Received February 21, 2018; accepted February 21, 2018; published online May 15, 2018

\begin{abstract}
Brucellosis is one of the most prevalent zoonoses in the world. Incidence of the disease has increased significantly in recent years and has seriously affected the health of human beings and the development of animal husbandry. The pathogenesis of brucellosis remains unclear. Current studies suggest that this disease may be related to changes in natural killer cells, dendritic cells, and macrophages in immune cell subsets. Brucellosis may be also related to $\mathrm{T}$ helper $(\mathrm{Th}) 1$ cell/Th2 cell imbalance in the $\mathrm{CD}^{+} \mathrm{T}$ cell subset, immunoregulation of regulatory T cells and Th17 cells, and the mechanism of action of $\mathrm{CD}^{+} \mathrm{T}$ cell. This paper aims to review the research progress on these inherent immune cells, the $\mathrm{CD} 4^{+} \mathrm{T}$ cell subset, and $\mathrm{CD} 8^{+} \mathrm{T}$ cells in Brucella infection.
\end{abstract}

Keywords: brucellosis, $\mathrm{CD}^{+} \mathrm{T}$ cell, CD8+ T cell, inherent immune cell

\section{Introduction}

Brucellosis is a zoonosis caused by Brucella [1]. Brucella is a genus of Gram- negative intracellular bacteria. The prevalent Brucella species in China mainly include three kinds, namely, B. abortus, B. ovis, and B. suis. Among these species, B. ovis features the strongest pathogenicity to humans [2-4]. People mainly become infected by Brucella by touching infected animals or ingesting contaminated meat and dairy products [5]. Brucella infection commonly manifests as fever, which is easily misdiagnosed as malaria, typhoid, rheumatic fever, osteoarthritis, and other diseases [6]. The pathogenesis of Brucella infection in humans remains unclear. In the presence of additional complications, diagnosis using epidemiological and clinical symptoms poses difficulties. Once it has entered into the chronic phase, curing the disease becomes impossible. Therefore, the pathogenesis of brucellosis is considered a research hot spot. Brucella infection is considered to be related to inherent immunity, adaptive immunity, and immunomodulatory mechanisms [7].

Papadopoulos et al. [8] have observed from in vitro implant bone model that Brucella can infect and replicate in bone marrow-derived dendritic cells (DCs) through granulocyte-macrophage colony-stimulating factor. Gao et al. [9] have discovered that when Brucella invades the host, the function of natural killer (NK) cells is inhibited, which can cause abortion. When type $1 \mathrm{~T}$ helper (Th1) cell is downregulated, the in vivo Th1 cell/Th2 cell imbalance can also cause the animal host to abort [10,11]. Th17 cells provide the host organism with additional defense against a number of extracellular and intracellular microbial infections and can defend against invasion of Brucella through cooperation with Th1 cells [12]. The antibody response to Brucella proteins, mediated by interleukin 17 (IL-17) secreted by Th17 cells, plays a key role in brucellosis vaccine [13]. Regulatory T cells (Tregs) inhibit the immune function of effector T cells, promoting Brucella invasion [14]. Therefore, the occurrence of brucellosis may be related to the imbalance in Treg/Th17 cells. Clapp et al. [15] have observed from nasal mucosa infection that when Brucella invades organisms through the nasal mucosa, $\mathrm{CD} 8^{+} \mathrm{T}$ cells play an important role in immune protection. Based on these research reports, we summarize the pathogenesis of inherent immune cells, as well as the role of $\mathrm{Th} 1 \mathrm{cell} / \mathrm{Th} 2$ cell in $\mathrm{CD} 4^{+} \mathrm{T}$ cell subsets, Treg and Th17 cells, and CD8 ${ }^{+} \mathrm{T}$ cells in brucellosis.

Department of Pharmacy, School of Medicine and Life Sciences, University of Jinan, Jinan 250200, China

*Correspondence: Jin Zhang, E-mail: jinzhangssd@126.com 


\section{Inherent immune cells and Brucella}

\subsection{NK cells and Brucella}

NK cells belong to a group of important inherent immune cells in organisms. They play an important role in the frontline defense system and participate in immunoregulation, as well as antitumor, anti-autoimmune disease and antiviral activities [16]. NK cells can directly dissolve Gram-negative bacteria and kill the target cells infected by microbes, thus playing an immunoregulatory role in $B$. abortus brucellosis [9]. The main cytokines that scavenge Brucella comprise tumor necrosis factor-alpha (TNF- $\alpha$ ), IL-6, IL-12, and interferon $\gamma$ (IFN- $\gamma$ ). Among these cytokines, IFN- $\gamma$ can activate the phagocytosis of macrophages, directly killing Brucella. Under stimulation by macrophages and the TNF- $\alpha$ secreted by NK cells, the process of phagocytosis for killing Brucella reaches the highest [9]. Jiménez et al. [17] have shown that in the case of lack of T cells and B cells, activation of NK cells serves as the critical factor for survival of Brucella-infected mouse. NK cells are activated in the host with brucellosis to participate in the control of propagation and diffusion of Brucella within the organism. To sum up, NK cells can be involved in scavenging Brucella by secreting TNF- $\alpha$ and other cytokines to confer immune protection on organisms.

\subsection{DCs, macrophages, and Brucella}

DCs include unique antigen-presenting cells with the strongest organism function and can activate resting $\mathrm{T}$ cells. DCs can efficiently take up, process, and present antigens. Immature DCs exhibit strong mobility, whereas the mature ones can effectively activate naïve T cells. DCs act as the central link in starting, regulating, and maintaining the immune response. When Brucella invades organisms, cyclic $\beta-1,2$-glucan in the bacterium seriously infects the DCs, causing the immune balance of $\mathrm{CD}^{+} \mathrm{T}$ cells to tend toward Th2 cell subsets. This condition can aid the invasion of pathogens [18]. Macrophages can identify and kill Brucella. However, the Brucella species that are not killed by macrophages continually reproduce in phagocytic cells. This condition damages macrophages and causes release of Brucella, which enters the body's circulation to cause bacteremia or septicemia. Ayarc1 et al. [19] have observed that when hosts are infected by Brucella, the level of high mobility group box 1 (HMGB1) in the serum increases, leading to septicemia and severe damages to the host. HMGB1 stimulates mononuclear cells and macrophages in the body to produce proinflammatory cytokines [20], including TNF- $\alpha$, IL-1 $\beta$, IL-6, and macrophage inflammatory protein-1 $\beta$, to increase the expression and release of various inflammatory molecules, resulting in aggravation and worsening of inflammation by brucellosis.

\section{$3 \mathrm{CD}^{+} \mathrm{T}$ cells and Brucella}

\subsection{Th1 cells/Th2 cells and Brucella}

$\mathrm{CD}^{+} \mathrm{T}$ cells play an important role in the immune process of resisting Brucella invasion. $\mathrm{CD}^{+} \mathrm{T}$ cells can activate the bactericidal functions of macrophages through secretion of IFN- $\gamma$ [21]. Dorneles et al. [22] have noted that after vaccinating cattle with Brucella, $\mathrm{CD}^{+}{ }^{+}$Th1 cells, induced by IFN- $\gamma$, participate in the initiation and development of control of Brucella, whereas $\mathrm{CD}^{+} \mathrm{T}$ cells, B cells, and Th2 cells exert no significant effects [23]. IFN- $\gamma$ secreted from Th1 cells plays an important role in the control of brucellosis. IFN- $\gamma$ can avoid utilizing the immune response induced by Th1, providing for development of a highly efficient vaccine to prevent brucellosis [24,25]. IFN- $\gamma$ secreted from Th1 cells and IL-4 secreted from Th2 cells are mutually antagonistic. IFN- $\gamma$ secreted from Th1 destroys the macrophages damaged by Brucella to provide immune protection to the organism [26]. Th1 cell/Th2 cell balance is very important in maintaining the normal immune response of organisms. Changes in Th1 cell/Th2 cell balance can cause various immune diseases, including autoimmune 
diseases, allergies, tumors, and infections [27]. Clausse et al. [28] used Brucella lumazine synthase and outer membrane protein 31 to stimulate the immune response of Th1 cells, Th2 cells, and mixed cells to generate IFN- $\gamma$ under adjuvant induction. Results indicated that the chimeric protein of Brucella can activate macrophages, induce the immune response mediated by Th1 cells, and confer protection to the body. Goel and Bhatnagar [29] evaluated the potential immunogenicity and protective effects of Brucella outer membrane protein $25 \mathrm{in} \mathrm{BALB} / \mathrm{c}$ mice and the toxic effects of brucellosis. These researchers showed increased immunoglobulin G (IgG)1/IgG2b ratio, as well as increased level of IL-4 in the mouse serum and increased immune response of Th2 cells. This condition caused an imbalance in the Th1 cell/Th2 cell ratio. Therefore, the increase in Th2 cells in the host may inhibit the immune response by Th1 cells. Imbalance in the Th1 cell/Th2 cell ratio results in the occurrence of brucellosis.

\subsection{Treg and Th17 cells and Brucella}

Treg is a kind of $\mathrm{CD}^{+} \mathrm{CD} 25^{+}$Foxp $^{+} \mathrm{T}$ cell subset with negatively regulatory function. This cell can play an immunosuppressive function through the release of IL-10, transforming growth factor $\beta$, and cytokines. IL-17 is the characteristic cytokine secreted by Th17 cells; it can resist invasion by pathogenic microbes, playing an important protective role in maintaining the immune homeostasis of organisms. IL-17 secreted by Th17 cells plays a core role in protective immunization against brucellosis [30]. Treg/Th17 balance plays an important role in controlling inflammation and pathogenesis through immune escape [31]. In cases of tumors, inflammatory responses, or autoimmune diseases, Treg/Th17 cell balance is destroyed, leading to a series of diseases. Wang et al. [32] have observed that in the body of aborted mice infected by Brucella, IL-10 secreted by Treg leads to functional imbalance between macrophages and the initial proinflammatory/anti-inflammatory cytokines, promoting the growth of and persistent infection by Brucella. Studies have shown that when the body of a sheep was immunized with Brucella M5-90 outer membrane protein, Treg exerted an immunosuppressive function. However, when Treg was downregulated, the immune response of IFN- $\gamma, \mathrm{CD}^{+} \mathrm{T}$ cell, and $\mathrm{CD}^{+} \mathrm{T}$ cell improved [32]. Experts speculated that brucellosis may be related to Treg/Th17 cell imbalance. However, in relation to the occurrence and development of the disease, the kind of immune mechanism induced by Treg/Th17 cell imbalance remains unclear. Thus, further studies should be conducted.

\section{$4 \mathrm{CDB}^{+} \mathrm{T}$ cell and Brucella}

Mature $\mathrm{T}$ lymphocytes can be divided into $\mathrm{CD} 4^{+} \mathrm{T}$ cells and $\mathrm{CD} 8^{+} \mathrm{T}$ cells, which play an important role in cellular immunity. Activated $\mathrm{CD}^{+}{ }^{+} \mathrm{T}$ cells differentiate into Th cells, which can enhance elimination of Brucella. Activated $\mathrm{CD}^{+} \mathrm{T}$ cells differentiate into cytotoxic $\mathrm{T}$ lymphocytes (CTL), which can dissolve macrophages infected by Brucella through perforin release to kill the organism intracellularly. Barrionuevo et al. [33] have observed from their study on the mechanism of B. abortus infection in organisms that invasion of Brucella can activate CTL, leading to an immune response in cells. When the immune function of the major histocompatibility complex class I is inhibited, $B$. abortus escapes the attack of CTL and undergoes immune escape, which further promotes chronic infection by brucellosis. Durward-Diioia et al. [34] have assumed that CD8 ${ }^{+}$ $\mathrm{T}$ cell failure inhibits the occurrence of IFN $-\gamma$ and the adaptive immune response, playing an important immune inhibition role in the chronic phase of brucellosis infection. Yin et al. [35] have discovered from a study on Brucella epitope vaccine that in BALB/c mice immune with recombinant protein, mixed Th1 cellsTh2 cell immune response mainly occurred. The ratio of $\mathrm{CD}^{+} \mathrm{T}$ cells $/ \mathrm{CD} 8^{+} \mathrm{T}$ cells and the expressions of $\operatorname{IgG}$ and IFN- $\gamma$ also increased. Attenuated brucellosis live vaccine M5-90 $\Delta$ wboa [36] is a potential vaccine for prophylaxis of the disease. This vaccine can activate $\mathrm{CD} 4^{+} \mathrm{T}$ cells and $\mathrm{CD} 8^{+} \mathrm{T}$ cells, can cause high expression of IFN- $\gamma$ in the body, and can provide desirable immune defense function. Therefore, if studies center on researching and developing a targeted vaccine that can activate $\mathrm{CD}^{+} \mathrm{T}$ cells in the early phase and $\mathrm{CD} 8^{+} \mathrm{T}$ cells in the late phase of brucellosis, the incidence of the disease may be significantly decreased. 


\section{Summary and prospects}

Brucellosis may be related to the immune regulatory function of inherent immune cells and $\mathrm{CD}^{+} \mathrm{T}$ cells, as well as imbalances in Th1 cells/Th2 cells and Treg/Th17 cells in CD4 ${ }^{+} \mathrm{T}$ subsets. However, the specific mechanism of the interaction of Treg/Th17 cells in brucellosis remains unclear and must be further investigated. With a wide range of epidemic areas and absence of typical clinical manifestations at the early stage, brucellosis is easily misdiagnosed and not easily eradicated upon entering into the chronic period. Therefore, prevention and control measures at the early stage of the disease bear importance. To prevent the occurrence of brucellosis, studies have focused on the development of vaccines. At present, the developed brucellosis vaccines mainly refer to attenuated live vaccines, including the following: S19, S2, M5, and RB51; inactivated vaccines, including 45/20, H38, 19-B, and 104M; mutant vaccines, including RB51, VTRM1 (rough mutant B. melitensis 16M), and VTRSl (rough mutant B. suis); and subunit vaccines, such as BLS-L7/L12. These vaccines enhance the corresponding immune response and perform protective roles. The scientific community is currently concentrated on studies of the immune mechanism of brucellosis to explore or to develop highpotency protective Brucella vaccines. Outbreak or prevalence of brucellosis in pastoral areas can be prevented upon development of a high-potency vaccine for brucellosis or discovery of a new method for regulating Th1 cell/Th2 cell balance and Treg/Th17 cell balance for brucellosis treatment.

Acknowledgments: None.

Conflict of interest: The author states no conflicts of interest.

\section{References}

[1] Golshani M., Rafati S., Dashti A., Gholami E., Siadat S.D., Oloomi M., et al., Vaccination with recombinant L7/L12-truncated Omp31 protein induces protection against Brucella infection in BALB/c mice, Mol. Immunol., 2015, 65(2), 287-292.

[2] Tian M., Qu J., Bao Y., Gao J., Liu J., Wang S., et al., Construction of pTM series plasmids for gene expression in Brucella species, J. Microbiol. Methods, 2016, 123, 18-23.

[3] Xiao Y., Zou G., Yin J., Tan W., Zhou J., Zhang H., Seroepidemiology of human Brucella infection in Yixing, China, Trop. Doct., 2017, 47(2), 165-167.

[4] Celli J., The changing nature of the Brucella-containing vacuole, Cell. Microbiol., 2015, 17(7), 951-958.

[5] Tumwine G., Matovu E., Kabasa J.D., Owiny D.O., Majalija S., Human brucellosis: sero-prevalence and associated risk factors in agro-pastoral communities of Kiboga District, Central Uganda, BMC Public Health, 2015, 15, 900, D0I: 10.1186/ s12889-015-2242-z.

[6] Assenga J.A., Matemba L.E., Muller S.K., Malakalinga J.J., Kazwala R.R., Epidemiology of Brucella infection in the human, livestock and wildlife interface in the Katavi-Rukwa ecosystem, Tanzania, BMC Vet. Res., 2015, 11, 189, DOI: 10.1186/ s12917-015-0504-8.

[7] Wang Z., Liu W., Wu T., Bie P., Wu Q., RNA-seq reveals the critical role of CspA in regulating Brucella melitensis metabolism and virulence, Sci. China Life Sci., 2016, 59(4), 417-424.

[8] Papadopoulos A., Gagnaire A., Degos C., De Chastellier C, Gorvel J.P., Brucella discriminates between mouse dendritic cell subsets upon in vitro infection, Virulence, 2016, 7(1), 33-44.

[9] Gao N., Jennings P., Guo Y., Yuan D., Regulatory role of natural killer (NK) cells on antibody responses to Brucella abortus, Innate Immun., 2011, 17(2), 152-163.

[10] Im Y.B., Park W.B., Jung M., Kim S., Yoo H.S., Evaluation of Th1/Th2 related immune response against recombinant proteins of Brucella abortus infection in mice, J. Microbiol. Biotechnol., 2016, 26(6), 1132-1139.

[11] Fahel J.S., de Souza M.B., Gomes M.T., Corsetti P.P., Carvalho N.B., Marinho F.A., et al., 5-Lipoxygenase negatively regulates Th1 response during Brucella abortus infection in mice, Infect. Immun., 2015, 83(3), 1210-1216.

[12] Clapp B., Skyberg J.A., Yang X., Thornburg T., Walters N., Pascual D.W., Protective live oral brucellosis vaccines stimulate Th1 and th17 cell responses, Infect. Immun., 2011, 79(10), 4165-4174.

[13] Abkar M., Lotfi A.S., Amani J., Eskandari K., Ramandi M.F., Salimian J., et al., Survey of Omp19 immunogenicity against Brucella abortus and Brucella melitensis: influence of nanoparticulation versus traditional immunization, Vet. Res. Commun., 2015, 39(4), 217-228. 
[14] Tian Z., Qiao J., Meng Q., Wang W., Chen C., Li C., et al., Dynamic changes of CD4+, CD8+ T, CD4+ CD25 + Treg in peripheral blood of sheep inoculated Brucella M5-90 attenuated vaccine, Chin. J. Prev. Vet. Med., 2013, 35(6), 504-506.

[15] Clapp B., Yang X., Thornburg T., Walters N., Pascual D.W., Nasal vaccination stimulates CD8 + T cells for potent protection against mucosal Brucella melitensis challenge, Immunol. Cell Biol., 2016, 94(5), 496-508.

[16] Mandal A., Viswanathan C., Natural killer cells: in health and disease, Hematol. Oncol. Stem Cell Ther., 2015, 8(2), 47-55.

[17] Jiménez de Bagüés M.P., de Martino A., Quintana J.F., Alcaraz A., Pardo J., Course of infection with the emergent pathogen Brucella microti in immunocompromised mice, Infect. Immun., 2011, 79(10), 3934-3939.

[18] Degos C., Gagnaire A., Banchereau R., Moriyón I., Gorvel J.P., Brucella C $\beta$ G induces a dual pro- and anti-inflammatory response leading to a transient neutrophil recruitment, Virulence, 2015, 6(1), 19-28.

[19] Ayarcı A.O., Yılmaz E., Sığırlı D., Budak F., Göral G., Oral H.B., Diagnostic value of serum concentrations of high-mobility group-box protein 1 and soluble hemoglobin scavenger receptor in brucellosis, Microbiol. Immunol., 2013, 57(2), 150-158.

[20] Cheng Y., Wang H., Mao M., Liang C., Zhang Y., Yang D., et al., Escin increases the survival rate of LPS-induced septic mice through inhibition of HMGB1 release from macrophages, Cell. Physiol. Biochem., 2015, 36(4), 1577-1586.

[21] Lacey C.A., Keleher L.L., Mitchell W.J., Brown C.R., Skyberg J.A., CXCR2 mediates Brucella-induced arthritis in interferon Y-deficient mice, J. Infect. Dis., 2016, 214(1), 151-160.

[22] Dorneles E.M., Lima G.K., Teixeira-Carvalho A., Araújo M.S., Martins-Filho O.A., Sriranganathan N., et al., Immune response of Calves vaccinated with Brucella abortus S19 or RB51 and revaccinated with RB51, PLoS ONE, 2015, 10(9), e0136696, DOI: 10.1371/journal.pone.0136696 eCollection2015.

[23] Vitry M.A., Hanot Mambres D, De Trez C, Akira S., Ryffel B., Letesson J.J., et al., Humoral immunity and CD4 + Th1 cells are both necessary for a fully protective immune response upon secondary infection with Brucella melitensis, J. Immunol., 2014, 192(8), 3740-3752.

[24] Coria L.M., Ibañez A.E., Pasquevich K.A., Cobiello P.L.G., Frank F.M., Giambartolomei G.H., et al., Brucella abortus Omp19 recombinant protein subcutaneously co-delivered with an antigen enhances antigen-specific $\mathrm{T}$ helper 1 memory responses and induces protection against parasite challenge, Vaccine, 2016, 34(4), 430-437.

[25] Pollak C.N., Wanke M.M., Estein S.M., Delpino M.V., Monachesi N.E., Comercio E.A., et al., Immunization with Brucella VirB proteins reduces organ colonization in mice through a Th1-type immune response and elicits a similar immune response in dogs, Clin. Vaccine Immunol., 2015, 22(3), 274-281.

[26] Zhang J., Yin S., Guo F., Meng R., Chen C., Zhang H., et al., A potent Brucella abortus 2308 ery live vaccine allows for the differentiation between natural and vaccinated infection, J. Microbiol., 2014, 52(8), 681-688.

[27] Xiang L., Rehm K.E., Marshall G.D. Jr., Effects of strenuous exercise on Th1/Th2 gene expression from human peripheral blood mononuclear cells of marathon participants, Mol. Immunol., 2014, 60(2), 129-134.

[28] Clausse M., Díaz A.G., Ghersi G., Zylberman V., Cassataro J., Giambartolomei G.H., et al., The vaccine candidate BLSOmp31 protects mice against Brucella canis infection, Vaccine, 2013, 31(51), 6129-6135.

[29] Goel D., Bhatnagar R., Intradermal immunization with outer membrane protein 25 protects BALB/c mice from virulent B.abortus 544, Mol. Immunol., 2012, 51(2), 159-168.

[30] Pasquevich K.A., Ibañez A.E., Coria L.M., García Samartino C, Estein S.M., Zwerdling A., et al., An oral vaccine based on $\mathrm{U}-0 \mathrm{mp} 19$ induces protection against $B$. abortus mucosal challenge by inducing an adaptive IL-17 immune response in mice, PLoS ONE, 2011, 6(1), e16203, DOI: 10.1371/journal.pone.0016203.

[31] Yan C., Zhang B.B., Hua H., Li B., Zhang B., Yu Q., et al., The dynamics of Treg/Th17 and the imbalance of Treg/Th17 in Clonorchis sinensis-infected mice, PLoS ONE, 2015, 10(11), e0143217, DOI: 10.1371/journal.pone. 0143217 eCollection 2015.

[32] Wang W., Wu J., Qiao J., Weng Y., Zhang H., Liao Q., et al., Evaluation of humoral and cellular immune responses to BP26 and OMP31 epitopes in the attenuated Brucella melitensis vaccinated sheep, Vaccine, 2014, 32(7), 825-833.

[33] Barrionuevo P., Delpino M.V., Pozner R.G., Velásquez L.N., Cassataro J., Giambartolomei G.H., Brucella abortus induces intracellular retention of MHC-I molecules in human macrophages down-modulating cytotoxic CD8 + T cell responses, Cell. Microbiol., 2013, 15(4), 487-502.

[34] Durward-Diioia M., Harms J., Khan M., Hall C., Smith J.A., Splitter G.A., CD8 + T cell exhaustion, suppressed gamma interferon production, and delayed memory response induced by chronic Brucella melitensis infection, Infect. Immun., 2015, 83(12), 4759-4771.

[35] Yin D., Li L., Song D., Liu Y., Ju W., Song X., et al., A novel recombinant multi-epitope protein against Brucella melitensis infection, Immunol. Lett., 2016, 175, 1-7.

[36] Li Z.Q., Shi J.X., Fu W.D., Zhang Y., Zhang J., Wang Z., et al., A Brucella melitensis M5-90 wboA deletion strain is attenuated and enhances vaccine efficacy, Mol. Immunol., 2015, 66(2), 276-283. 